\title{
EIGENVALUE PROBLEMS IN A NON-LIPSCHITZ DOMAIN
}

\author{
GABRIEL ACOSTA AND MARÍA G. ARMENTANO
}

\begin{abstract}
In this paper we analyze piecewise linear finite element approximations of the Laplace eigenvalue problem in a plane domain with an external cusp. Since the domain under consideration is curved and non-Lipschitz, the classical spectral theory can not be applied directly. We present the eigenvalue problem in a proper setting, and relying on known convergence results for the associated source problem, we obtain quasi optimal order of convergence for the eigenpairs.
\end{abstract}

\section{INTRODUCTION}

The numerical approximation of eigenvalues and eigenfunctions by the finite element method is a major concern in science and engineering and has been widely analyzed from the theoretical point of view for different kind of spectral problems. In the context of compact operators a general and well known theory $[4,5,11]$ allows to obtain, under appropriate assumptions, spectral convergence of a discrete approximation from convergence results of the source counterpart. This is particularly true in conforming methods for problems involving polygonal domains since, in general, them fit straightforwardly the framework given by the general theory. On the contrary, for a curved domain $\Omega$ and linear elements, the convergence of the method for the source problem does not guarantee the convergence of the spectral approximation, since the domain $\Omega_{h}$ given by the finite element mesh does not agree with $\Omega$. Indeed, as it is clearly pointed out in the literature, even for the Laplace operator and smooth domains, the eigenvalue approximation problem needs a particular treatment for both, Dirichlet $[9,12,13,14]$ as well as Neumann [7] boundary conditions.

The goal of this paper is the analysis of piecewise linear finite element spectral approximations of the Laplace operator with mixed boundary conditions, in a plane domain with an external cusp. This problem is not covered by the known literature which, as far as we know, only deals with polygonal or smooth domains.

Let $\Omega \subset \mathbb{R}^{2}$ be:

$$
\Omega=\left\{(x, y): 0<x<1,0<y<x^{\alpha}\right\},
$$

where $\alpha>1$. With $\Gamma=\Gamma_{1} \cup \Gamma_{2} \cup \Gamma_{3}$ we denote the boundary of $\Omega$, where

$$
\Gamma_{1}=\{0 \leq x \leq 1, y=0\}, \quad \Gamma_{2}=\{x=1,0 \leq y \leq 1\} \text { and } \Gamma_{3}=\left\{0 \leq x \leq 1, y=x^{\alpha}\right\}
$$

(see Figure 1).

1991 Mathematics Subject Classification. 65N25, 65N30.

Key words and phrases. Cuspidal domains, Eigenvalue problems, Finite elements, Graded meshes.

Supported by ANPCyT, under grants PICT 2007-00910 and PICT 2010- 01675, and by Universidad de Buenos Aires under grant 20020100100143. 


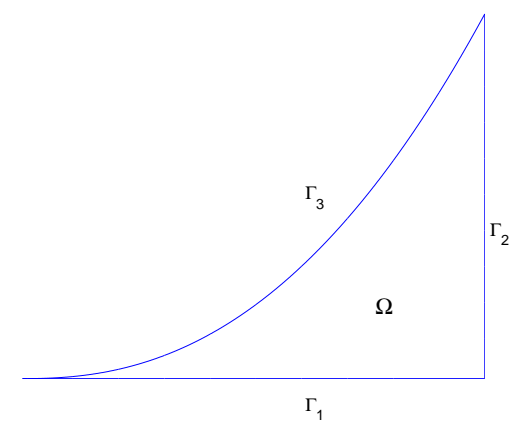

Figure 1. Cuspidal domain

We consider the following eigenvalue problem:

$$
\left\{\begin{aligned}
-\Delta u & =\lambda u, & & \text { in } \Omega \\
\frac{\partial u}{\partial \nu} & =0, & & \text { on } \Gamma_{1} \cup \Gamma_{3} \\
u & =0, & & \text { on } \Gamma_{2}
\end{aligned}\right.
$$

where $\nu$ denotes the outside normal.

Taking $V=\left\{v \in H^{1}(\Omega):\left.v\right|_{\Gamma_{2}}=0\right\}$, the variational formulation of (1.1) is given by: find $\lambda \in \mathbb{R}$ and $u \in V, u \neq 0$ such that

$$
a(u, v)=\lambda b(u, v) \quad \forall v \in V
$$

where $a(u, v)=\int_{\Omega} \nabla u \nabla v$ and $b(u, v)=\int_{\Omega} u v$.

In order to introduce the linear finite element approximation of (1.2) we consider a family of triangulations $\left\{\mathcal{T}_{h}\right\}$, and we write $\Omega_{h}=\cup_{T \in \mathcal{T}_{h}} T$. We assume that the nodes belonging to $\Gamma_{h}$, the boundary of $\Omega_{h}$, also belong to $\Gamma$, and therefore $\Omega \subset \Omega_{h}$. Observe that each $\mathcal{T}_{h}$ is actually a triangulation of $\Omega_{h}$ instead of a triangulation of $\Omega$.

Associated with $\left\{\mathcal{T}_{h}\right\}$ we have the finite element space

$$
V_{h}=\left\{v \in H^{1}\left(\Omega_{h}\right):\left.v\right|_{\Gamma_{2}}=0 \text { and }\left.v\right|_{T} \in \mathcal{P}_{1} \quad \forall T \in \mathcal{T}_{h}\right\}
$$

where $\mathcal{P}_{1}$ denotes the space of linear polynomials.

Then, the finite element approximation problem of (1.2) reads: find $\lambda_{h} \in \mathbb{R}$ and $u_{h} \in V_{h}$, $u_{h} \neq 0$ satisfying

$$
a_{h}\left(u_{h}, v_{h}\right)=\lambda_{h} b_{h}\left(u_{h}, v_{h}\right) \quad \forall v_{h} \in V_{h}
$$

where $a_{h}(u, v)=\int_{\Omega_{h}} \nabla u \nabla v$ and $b_{h}(u, v)=\int_{\Omega_{h}} u v$.

In order to analyze the convergence properties of (1.3) we use the abstract approximation theory stated in $[4,5,11]$. Therefore we need to introduce appropriate compact operators $T$ and $T_{h}$ associated to (1.2) and (1.3) respectively defined in the same functional space (a requirement not easy to fulfill since $\Omega \neq \Omega_{h}$ ) and such that $T_{h} \rightarrow T$ in norm. In the case of a smooth curved domain $\Omega$, and the Laplace spectral problem with Neumann boundary conditions, in [7] the authors successfully define $T$ and $T_{h}$ in $L^{2}(\Omega)$ and obtain optimal order of convergence for the eigenpairs. Since in our problem the Dirichlet data is given only on the straight parts of $\Gamma$, the method described in [7] can be easily adapted to our setting. However, following that approach we were not able to obtain optimal order of convergence for eigenvalues nor for eigenfunctions in $H^{1}$, since certain classical results in Sobolev spaces do not hold in domains with external cusps. Therefore, we propose a different approach and define $T$ and $T_{h}$ in $L^{2}\left(\mathbb{R}^{2}\right)$ extending by zero the involved functions. Due to the existence of Neumann boundary conditions this procedure is not 
standard and, as expected, only provides a poor order of convergence in $L^{2}$. Nevertheless, by means of a further analysis, we prove optimal order of convergence for eigenvalues and optimal order error estimates for eigenfunctions in the $H^{1}$ norm.

Let us recall that the Poisson's source problem associated to (1.1) plays a fundamental role in the analysis of convergence of the spectral discrete approximation $[4,5,11]$. In this regard it is shown in $[2,8]$ that the solution of such a problem belongs to $H^{2}(\Omega)$ if the source $f$ is in $L^{2}(\Omega)$ and if $1<\alpha<3$. Moreover, quasi-optimal order error estimates in $L^{2}$ and $H^{1}$ were respectively obtained in [1] and [3] for linear finite element approximations using appropriated graded meshes.

This paper is organized as follows: In Section 2 we introduce linear operators $T$ and $T_{h}$ associated to the problems (1.2) and (1.3) respectively. In Section 3 we recall some results involving the graded meshes that we will use in the rest of the paper. In Section 4, we prove convergence of the eigenfunctions and eigenvalues with quasi optimal order. Finally, in Section 5 , we show some numerical examples and we study the behavior of the discrete approximation of the first eigenvalue that seems to be monotonically decreasing when $h$ goes to zero.

\section{Statement of the SPeCtral Problems}

In this section we introduce linear operators $T$ and $T_{h}$ with spectra related to those of problems (1.2) and (1.3) respectively, and in Section 4 we show that $T_{h} \rightarrow T$ in norm when $h$ goes to zero.

For a function $w$ defined in some domain $\mathcal{D} \subset \mathbb{R}^{2}$, we denote with $\bar{w}$ its extension by zero from $\mathcal{D}$ to $\mathbb{R}^{2}$.

Let $T$ be the operator defined by

$$
T: L^{2}\left(\mathbb{R}^{2}\right) \rightarrow L^{2}\left(\mathbb{R}^{2}\right) \quad T f=\bar{u},
$$

with $u \in V$ the solution of

$$
a(u, v)=b(f, v) \quad \forall v \in V .
$$

It is known that the solution of $(2.4)$ is in $H^{2}(\Omega)$ if the source $f$ is in $L^{2}(\Omega)$ and if $1<\alpha<3$ $[2,6,8]$. Moreover, there exists a constant $C$ such that

$$
\|u\|_{H^{2}(\Omega)} \leq C\|f\|_{L^{2}(\Omega)}
$$

In particular, $T$ is a well-defined bounded self adjoint operator

$$
\|T f\|_{L^{2}\left(\mathbb{R}^{2}\right)} \leq C\|f\|_{L^{2}\left(\mathbb{R}^{2}\right)} .
$$

It is easy to see that $\lambda_{j}$ is a positive eigenvalue of (1.2) if and only if $\mu_{j}:=\frac{1}{\lambda_{j}}$ is a positive eigenvalue of the operator $T$ and the corresponding eigenfunctions are related by $u_{j} \longleftrightarrow \bar{u}_{j}$. Therefore, from the regularity results and the classical spectral theory we can conclude that the eigenfunctions $u_{j}$ lies in $H^{2}(\Omega)$. The a priori estimate (2.5) together with compact imbedding results for external cusps (see page 430 in [10]) show that $T$ is a compact operator. Its spectral properties as well as the spectral approximation theory relies on this fact. On the other hand, even when $\Omega$ is not regular, some extension operator can be constructed. More precisely, the solution of (1.2) can be extended to a function in a weighted Sobolev space with the weight being a power of the distance to the cuspidal point $[3,10]$. Indeed, there exists a function $u^{E} \in H_{\alpha}^{2}\left(\mathbb{R}^{2}\right)$ such that $\left.u^{E}\right|_{\Omega}=u$, and

$$
\left\|u^{E}\right\|_{H_{\alpha}^{2}\left(\mathbb{R}^{2}\right)} \leq C\|u\|_{H^{2}(\Omega)} .
$$

where the weighted Sobolev space $H_{\alpha}^{2}$ is defined, for any domain $\mathcal{D} \subset \mathbb{R}^{2}$, as follows:

$$
H_{\alpha}^{2}(\mathcal{D})=\left\{v: r^{\frac{\alpha-1}{2}} D^{\gamma} v \in L^{2}(\mathcal{D}) \quad \forall \gamma,|\gamma| \leq 2\right\}
$$


with $r=\sqrt{x^{2}+y^{2}}$, and

$$
\|v\|_{H_{\alpha}^{2}(\mathcal{D})}^{2}=\sum_{|\gamma| \leq 2}\left\|r^{\frac{\alpha-1}{2}} D^{\gamma} v\right\|_{L^{2}(\mathcal{D})}^{2} .
$$

This extension result will be useful for bounding the aproximation error.

Now, we define the discrete analogue of the operator $T$ as:

$$
T_{h}: L^{2}\left(\mathbb{R}^{2}\right) \rightarrow L^{2}\left(\mathbb{R}^{2}\right) \quad T_{h} f=\bar{u}_{h}
$$

with $u_{h}$ is the solution of

$$
a_{h}\left(u_{h}, v_{h}\right)=b_{h}\left(f, v_{h}\right) \quad \forall v_{h} \in V_{h}
$$

Using the Poincaré inequality, it is easy to see that the bilinear form $a_{h}(\cdot, \cdot)$ is coercive and continuous on $V_{h}$ and the linear functional $L_{h}(v)=b_{h}(f, v)$ is continuous on $V_{h}$. Therefore, from the Lax-Milgram theorem, we can conclude that there exists a unique solution $u_{h} \in V_{h}$ of problem (2.8). So, the operator $T_{h}$ is well defined and there exits a constant $C$, independent of $h$, such that

$$
\left\|T_{h} f\right\|_{L^{2}\left(\mathbb{R}^{2}\right)} \leq C\|f\|_{L^{2}\left(\mathbb{R}^{2}\right)}
$$

It is easy to see, as in the continuous case, that $\lambda_{h . j}$ is a positive eigenvalue of (1.3) if and only if $\mu_{h, j}$ is a positive eigenvalue of the operator $T_{h}$ and the corresponding eigenfunctions $u_{h, j}$, $\bar{u}_{h, j}$ satisfies $u_{h, j}=\left.\bar{u}_{h, j}\right|_{\Omega}$.

In order to prove that the operators $T_{h}$ converge to $T$ in norm when $h$ goes to zero, we need to analyze the error between the solutions of the problems (2.4) and (2.8).

\section{Graded Meshes}

We will assume that the family of meshes $\left\{\mathcal{T}_{h}\right\}$ satisfies the same properties considered in $[1,3]$. More precisely, we take $1<\alpha<3$ and define $\gamma=(\alpha-1) / 2$. Let $\mathcal{T}_{h}$ be a triangulation of $\Omega_{h}$, where $\Omega_{h}$ is an approximate polygon of $\Omega$ with all its vertices belonging to $\Gamma$, and $h>0$ be a parameter that goes to 0 . If for each $T \in \mathcal{T}_{h}$ we denote by $h_{T}$ its diameter and by $\theta_{T}$ its maximum angle, we assume that there exist positive constants $\sigma$ and $\theta_{M}<\pi$, independent of $h$, such that

(1) $\theta_{T}<\theta_{M}, \forall T \in \mathcal{T}_{h}$ (the maximum angle condition).

(2) $h_{T} \sim \sigma h^{\frac{1}{1-\gamma}}$, if $(0,0) \in T$.

(3) $h_{T} \leq \sigma h \inf _{T} x^{\gamma}$, if $(0,0) \notin T$.

We denote by $\Gamma_{3, h}^{j}, 1 \leq j \leq n$, the edges on the boundary of $\Omega_{h}$, by $P_{j-1}=\left(x_{j-1}, x_{j-1}^{\alpha}\right)$ and $P_{j}=\left(x_{j}, x_{j}^{\alpha}\right)$ their endpoints with $x_{0}=0$ and $x_{n}=1$, and by $\Gamma_{3}^{j}$ the part on $\Gamma_{3}$ with the same endpoints (see Figure 2). By $\Omega_{h}^{j}$ we denote the region bounded by $\Gamma_{3}^{j}$ and $\Gamma_{3, h}^{j}$.

In addition to the assumptions (1), (2) and (3) we will need for our error analysis the following hypothesis on the meshes:

(Ha) For $1 \leq j \leq n$ the region $\Omega_{h}^{j}$ is strictly contained in only one triangle denoted by $T_{j}$. We denote the diameter of $T_{j}$ by $h_{j}$.

Let us also notice that, for $2 \leq j \leq n$,

$$
x_{j} \leq C x_{j-1}
$$




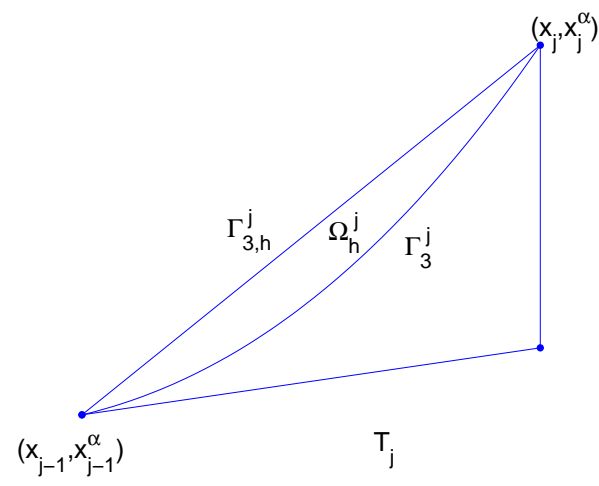

Figure 2

where $C$ can be taken independent of $h$. Indeed, from (Ha) we have $x_{j}-x_{j-1} \leq C\left|\Gamma_{3, h}^{j}\right|$ for some constant depending only on $\alpha$. Then, $x_{j}-x_{j-1} \leq C h_{j}$, and hence, from assumption (3) we get

$$
x_{j} \leq x_{j-1}\left(1+C h x_{j-1}^{\gamma-1}\right) .
$$

Therefore, we have proved the following useful result

Lemma 3.1. For $2 \leq j \leq n$,

$$
x_{j-1} \leq x_{j} \leq C x_{j-1}
$$

with $C$ depending only on $\alpha$ and $\sigma$.

We also have, for these graded meshes, the following bound for the measure of the set $\Omega_{h} \backslash \Omega$ in terms of the parameter $h$ (see [1]):

$$
\left|\Omega_{h} \backslash \Omega\right| \leq C h^{2} .
$$

We will need, in addition to assumptions (1),(2),(3) and (Ha), the following hypothesis about the mesh:

(Hb) For each triangle $T_{j}$ with vertices $P_{j-1}, P_{j}, R_{j}$, and for $h_{j}$ small enough, the triangle $\tilde{T}_{j}$ with vertices $\frac{P_{j-1}+R_{j}}{2}, \frac{P_{j}+R_{j}}{2}, R_{j}$ (see Figure 3) does not intersect $\Omega_{h}^{j}$.

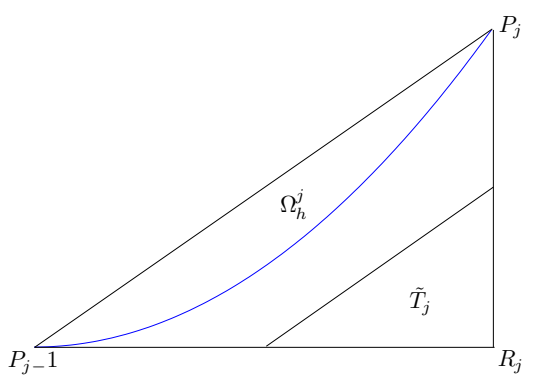

Figure 3

Remark 3.1. As we have mentioned in [1] it can easily be deduced that (Hb) holds for meshes with only regular elements, and for domains with smooth boundaries. Meshes for the domains under consideration in this paper involve necessarily anisotropic elements and this kind of elements may fail to verify condition ( $\mathrm{Hb})$. 
In what follows we will assume that the family of triangulations under consideration verifies $(1),(2),(3),(\mathrm{Ha})$ and $(\mathrm{Hb})$.

\section{Spectral approximation}

In the next lemma we show that $T_{h} \rightarrow T$ in norm as $h$ goes to zero. Despite the poor order given in (4.10) this result allows us to state the convergence for eigenvalues.

Lemma 4.1. There exists a positive constant $C$ such that for any $f \in L^{2}\left(\mathbb{R}^{2}\right)$

$$
\left\|T f-T_{h} f\right\|_{L^{2}\left(\mathbb{R}^{2}\right)} \leq C h\|f\|_{L^{2}\left(\mathbb{R}^{2}\right)}
$$

Proof. For any $f \in L^{2}\left(\mathbb{R}^{2}\right)$ from the definition of the operators $T$ and $T_{h}$ we have that

$$
\left\|T f-T_{h} f\right\|_{L^{2}\left(\mathbb{R}^{2}\right)}^{2}=\left\|\bar{u}-\bar{u}_{h}\right\|_{L^{2}\left(\mathbb{R}^{2}\right)}^{2}\|=\| \bar{u}-u_{h}\left\|_{L^{2}\left(\Omega_{h}\right)}^{2}=\right\| u-u_{h}\left\|_{L^{2}(\Omega)}^{2}+\right\| u_{h} \|_{L^{2}\left(\Omega_{h} \backslash \Omega\right)}^{2}
$$

From the $L^{2}$ error estimates given in Theorem 4.2 in [1] we get

$$
\left\|T f-T_{h} f\right\|_{L^{2}\left(\mathbb{R}^{2}\right)}^{2} \leq C h^{4} \log ^{2}(1 / h)\|f\|_{L^{2}(\Omega)}^{2}+C h^{2}\|f\|_{L^{2}\left(\Omega_{h} \backslash \Omega\right)}^{2}+\left\|u_{h}\right\|_{L^{2}\left(\Omega_{h} \backslash \Omega\right)}^{2}
$$

and hence we only need to bound the last term of the previous inequality. Since,

$$
\left\|u_{h}\right\|_{L^{2}\left(\Omega_{h} \backslash \Omega\right)}^{2} \leq 2\left\|u^{E}-u_{h}\right\|_{L^{2}\left(\Omega_{h} \backslash \Omega\right)}^{2}+2\left\|u^{E}\right\|_{L^{2}\left(\Omega_{h} \backslash \Omega\right)}^{2}
$$

by using Theorem 5.1 in [1] we obtain

$$
\left\|u_{h}\right\|_{L^{2}\left(\Omega_{h} \backslash \Omega\right)}^{2} \leq C h^{4} \log ^{2}(1 / h)\|f\|_{L^{2}(\Omega)}^{2}+C h^{2}\|f\|_{L^{2}\left(\Omega_{h} \backslash \Omega\right)}^{2}+2\left\|u^{E}\right\|_{L^{2}\left(\Omega_{h} \backslash \Omega\right)}^{2}
$$

From (4.4) in [3] we know that $u^{E} \in L^{\infty}\left(\Omega_{h}\right)$ and there exists a constant $C$, independent of $h$ and $\alpha$, such that

$$
\left\|u^{E}\right\|_{L^{\infty}\left(\Omega_{h}\right)} \leq C\|u\|_{H^{2}(\Omega)}
$$

Therefore, from (3.9) we obtain

$$
\left\|u^{E}\right\|_{L^{2}\left(\Omega_{h} \backslash \Omega\right)} \leq C\left|\Omega_{h} \backslash \Omega\right|^{\frac{1}{2}}\|u\|_{H^{2}(\Omega)} \leq C h\|u\|_{H^{2}(\Omega)}
$$

and using (2.5) we get

and so

$$
\left\|u^{E}\right\|_{L^{2}\left(\Omega_{h} \backslash \Omega\right)} \leq C h\|f\|_{L^{2}(\Omega)}
$$

$$
\left\|u_{h}\right\|_{L^{2}\left(\Omega_{h} \backslash \Omega\right)}^{2} \leq C h^{4} \log ^{2}(1 / h)\|f\|_{L^{2}(\Omega)}^{2}+C h^{2}\|f\|_{L^{2}\left(\Omega_{h} \backslash \Omega\right)}^{2}+C h^{2}\|f\|_{L^{2}(\Omega)}
$$

and the proof concludes from (4.11) and the previous estimate.

Remark 4.1. We observe that if $f$ is an eigenfunction of $T$ then $f \equiv 0$ outside of $\Omega$, and therefore by using Theorem 3.2 in [1], we get

$$
\left\|T f-T_{h} f\right\|_{L^{2}\left(\mathbb{R}^{2}\right)}^{2} \leq C h^{4} \log ^{2}(1 / h)\|f\|_{L^{2}(\Omega)}^{2}+\left\|u_{h}\right\|_{L^{2}\left(\Omega_{h} \backslash \Omega\right) .}^{2}
$$

In view of (4.13) the order in $L^{2}$ norm can not be improved with our approach.

Now, we apply the spectral approximation theory given in [4].

Let $\mu$ be an eigenvalue of $T$ with multiplicity $m$, we denote by $\mathcal{E}$ the associated eigenspace. From Lemma 4.1 we know that $T_{h}$ converges to $T$ in norm when $h$ goes to 0 and so, there exist exactly $m$ eigenvalues of $T_{h}, \mu_{h}^{1}, \cdots, \mu_{h}^{m}$ repeated according to their algebraic multiplicities converging to $\mu$. Let $\mathcal{E}_{h}$ be the direct sum of the associated eigenspaces. Defining the gap $\bar{\delta}$ between two subspaces, $\mathcal{X}$ and $\mathcal{Y}$ of $L^{2}\left(\mathbb{R}^{2}\right)$

$$
\bar{\delta}(\mathcal{X}, \mathcal{Y}):=\max \{\delta(\mathcal{X}, \mathcal{Y}), \delta(\mathcal{Y}, \mathcal{X})\}
$$


with

$$
\delta(\mathcal{X}, \mathcal{Y})=\sup _{v \in \mathcal{X},\|v\|_{L^{2}\left(\mathbb{R}^{2}\right)}=1}\left(\inf _{w \in \mathcal{Y}}\|v-w\|_{L^{2}\left(\mathbb{R}^{2}\right)}\right)
$$

we have the following result

Lemma 4.2. There exists a constant $C$ such that

$$
\bar{\delta}\left(\mathcal{E}, \mathcal{E}_{h}\right) \leq C h
$$

Proof. It is a consequence of Lemma 4.1, and Theorem 7.1 in [4].

From the spectral theory it follows immediately that (see, for example, Theorem 2 in [11])

$$
\left|\mu_{j}-\mu_{h, j}\right| \leq C h
$$

Corollary 4.1. Let $\mu_{j}$ be the $j$-th (simple) eigenvalue of $T$ and let $\mu_{h, j}$ be the associated $j$-th eigenvalue of $T_{h}$. Then, the corresponding eigenfunctions $u_{j}$ and $u_{h, j}$ can be chosen such that $\left\|u_{j}\right\|_{L^{2}(\Omega)}=1,\left\|u_{h, j}\right\|_{L^{2}(\Omega)}=1$ and

$$
\left\|u_{j}-u_{h, j}\right\|_{L^{2}(\Omega)} \leq C h,
$$

with $C$ a strictly positive constant.

Proof. From the spectral theory we know that, given $\mu_{j}$ the $j$-th (simple) eigenvalue of $T$ and $\mu_{h, j}$ the associated $j$-th eigenvalue of $T_{h}$ the corresponding eigenfunctions $\bar{u}_{j}$ and $\bar{u}_{h, j}$ can be chosen in such a way that $\left\|\bar{u}_{j}\right\|_{L^{2}\left(\mathbb{R}^{2}\right)}=\left\|u_{j}\right\|_{L^{2}(\Omega)}=1,\left\|\bar{u}_{h, j}\right\|_{L^{2}\left(\mathbb{R}^{2}\right)}=\left\|u_{h, j}\right\|_{L^{2}\left(\Omega_{h}\right)}=1$ and

$$
\left\|u_{j}-u_{h, j}\right\|_{L^{2}(\Omega)} \leq\left\|\bar{u}_{j}-\bar{u}_{h, j}\right\|_{L^{2}\left(\mathbb{R}^{2}\right)} \leq C h .
$$

Now, let $\hat{u}_{h, j}=\bar{u}_{h, j} /\left\|\bar{u}_{h, j}\right\|_{L^{2}(\Omega)}$ then, since

$$
\left\|u_{h, j}\right\|_{L^{2}(\Omega)}^{2}=\left\|u_{h, j}\right\|_{L^{2}\left(\Omega_{h}\right)}^{2}-\left\|u_{h, j}\right\|_{L^{2}\left(\Omega_{h} \backslash \Omega\right)}^{2}=1-\left\|u_{h, j}\right\|_{L^{2}\left(\Omega_{h} \backslash \Omega\right)}^{2},
$$

from (4.15) and (4.13) we get

$$
\begin{aligned}
\left\|u_{j}-\hat{u}_{h, j}\right\|_{L^{2}(\Omega)} & \leq\left\|u_{j}-u_{h, j}\right\|_{L^{2}(\Omega)}+\left\|u_{h, j}\left(1-\frac{1}{\left\|u_{h, j}\right\|_{L^{2}(\Omega)}}\right)\right\|_{L^{2}(\Omega)} \\
& \leq C h+\left|1-\frac{1}{\left\|u_{h, j}\right\|_{L^{2}(\Omega)}}\right|=C h+\frac{1-\left\|u_{h, j}\right\|_{L^{2}(\Omega)}^{2}}{\left\|u_{h, j}\right\|_{L^{2}(\Omega)}\left(1+\left\|u_{h, j}\right\|_{L^{2}(\Omega)}\right)} \\
& \leq C h+\frac{1-\left\|u_{h, j}\right\|_{L^{2}(\Omega)}^{2}}{\left\|u_{h, j}\right\|_{L^{2}(\Omega)}^{2}}=C h+\frac{\left\|u_{h, j}\right\|_{L^{2}\left(\Omega_{h} \backslash \Omega\right)}^{2}}{\left\|u_{h, j}\right\|_{L^{2}(\Omega)}^{2}} \\
& \leq C h+\frac{C h^{2}}{1-C h^{2}},
\end{aligned}
$$

and we conclude the proof.

The order for the approximate eigenvalues can be improved by using the following lemma.

Lemma 4.3. There exists a positive constant $C$ such that

$$
\left|\int_{\mathbb{R}^{2}}\left(T-T_{h}\right) f g\right| \leq C h^{2} \log (1 / h)\|f\|_{L^{2}\left(\mathbb{R}^{2}\right)}\|g\|_{L^{2}\left(\mathbb{R}^{2}\right)} \quad \forall f, g \in \mathcal{E}
$$


Proof. Let $f, g \in \mathcal{E}$ and let

$$
\begin{array}{cccc}
\bar{u}=T f, & u=\left.\bar{u}\right|_{\Omega}, & \bar{u}_{h}=T_{h} f, & u_{h}=\left.\bar{u}_{h}\right|_{\Omega} \\
\bar{v}=T g, & v=\left.\bar{v}\right|_{\Omega_{h}}, & \bar{v}_{h}=T_{h} g, & v_{h}=\left.\bar{v}_{h}\right|_{\Omega_{h}}
\end{array}
$$

Then, since $\left.f\right|_{\mathbb{R}^{2} \backslash \Omega}=0$ and $\left.g\right|_{\mathbb{R}^{2} \backslash \Omega}=0$ we have that

$$
\int_{\mathbb{R}^{2}}\left(T-T_{h}\right) f g=\int_{\Omega}\left(T-T_{h}\right) f g=\int_{\Omega}\left(\bar{u}-\bar{u}_{h}\right) g=\int_{\Omega} u g-\int_{\Omega} u_{h} g
$$

On the other hand, from (2.4) and (2.8), we have that

and

$$
\begin{aligned}
& \int_{\Omega} \nabla u \nabla w=\int_{\Omega} f w \quad \forall w \in V \\
& \int_{\Omega} \nabla v \nabla w=\int_{\Omega} g w \quad \forall w \in V
\end{aligned}
$$

$$
\begin{gathered}
\int_{\Omega_{h}} \nabla u_{h} \nabla w_{h}=\int_{\Omega} f w_{h} \quad \forall w_{h} \in V_{h} \\
\int_{\Omega_{h}} \nabla v_{h} \nabla w_{h}=\int_{\Omega} g w_{h} \quad \forall w_{h} \in V_{h}
\end{gathered}
$$

In particular,

$$
\begin{aligned}
\int_{\Omega} u g & =\int_{\Omega} \nabla u \nabla v \\
\int_{\Omega} u_{h} g & =\int_{\Omega_{h}} \nabla v_{h} \nabla u_{h}
\end{aligned}
$$

and, since functions in $V_{h}$ restricted to $\Omega$ belong to $V$, we also have that

and

$$
\int_{\Omega_{h}} \nabla u_{h} \nabla v_{h}=\int_{\Omega} f v_{h}=\int_{\Omega} \nabla u \nabla v_{h}
$$

$$
\int_{\Omega_{h}} \nabla v_{h} \nabla u_{h}=\int_{\Omega} g u_{h}=\int_{\Omega} \nabla v \nabla u_{h} .
$$

As a consequence,

$$
\begin{aligned}
\int_{\Omega} u g-\int_{\Omega} u_{h} g & =\int_{\Omega} u g-\int_{\Omega} \nabla u \nabla v_{h}-\int_{\Omega} \nabla u_{h} \nabla v+\int_{\Omega_{h}} \nabla u_{h} \nabla v_{h} \\
& =\int_{\Omega} \nabla\left(u-u_{h}\right) \nabla\left(v-v_{h}\right)+\int_{\Omega_{h} \backslash \Omega} \nabla u_{h} \nabla v_{h}
\end{aligned}
$$

For the first term in (4.16), by Theorem 4.1 in [1], we know that

$$
\int_{\Omega} \nabla\left(u-u_{h}\right) \nabla\left(v-v_{h}\right) \leq C h^{2} \log (1 / h)
$$

On the other hand, we have that

$$
\left\|\nabla u_{h}\right\|_{L^{2}\left(\Omega_{h} \backslash \Omega\right)} \leq\left\|\nabla u^{E}-\nabla u_{h}\right\|_{L^{2}\left(\Omega_{h} \backslash \Omega\right)}+\left\|\nabla u^{E}\right\|_{L^{2}\left(\Omega_{h} \backslash \Omega\right)} .
$$

Then, this estimates joint with Lemma 2.7 and Theorem 4.1 in [1] and (2.5) allows us to conclude that

$$
\left\|\nabla u_{h}\right\|_{L^{2}\left(\Omega_{h} \backslash \Omega\right)} \leq C h \sqrt{\log (1 / h)}\|f\|_{L^{2}(\Omega)}
$$

Hence, in view that an analogous estimate for $v_{h}$ holds, we conclude the proof. 
Now we are able to establish the optimal order error estimate for the approximate eigenvalues.

Theorem 4.1. Let $\mu_{j}$ be the $j$-th (simple) eigenvalue of $T$ and $\mu_{h, j}$ the $j$-th eigenvalue of $T_{h}$. Then, there exists a strictly positive constant $C$ such that

$$
\left|\mu_{j}-\mu_{h, j}\right| \leq C h^{2} \log (1 / h)
$$

Proof. It follows from the previous lemma, Lemma 4.1, and Theorem 7.3 in [4].

The next lemma gives an expression for the difference between the $j$-th eigenvalue $\lambda_{j}$ and its approximation $\lambda_{h, j}$ and it is, in particular, a tool to obtain optimal order error estimates for eigenfunctions in $H^{1}$ norm.

Lemma 4.4. Let $\left(\lambda_{j}, u_{j}\right)$ and $\left(\lambda_{h, j}, u_{h, j}\right)$ be the $j$-th eigenpairs solutions of problems (1.2) and (1.3) respectively, with $\left\|u_{j}\right\|_{L^{2}(\Omega)}=1$ and $\left\|u_{h, j}\right\|_{L^{2}(\Omega)}=1$. Then we have that

$$
\lambda_{h, j}-\lambda_{j}=\left\|\nabla\left(u_{j}-u_{h, j}\right)\right\|_{L^{2}(\Omega)}^{2}-\lambda_{j}\left\|u_{j}-u_{h, j}\right\|_{L^{2}(\Omega)}^{2}+\left\|\nabla u_{h, j}\right\|_{L^{2}\left(\Omega_{h} \backslash \Omega\right)}^{2}-\lambda_{h, j}\left\|u_{h, j}\right\|_{L^{2}\left(\Omega_{h} \backslash \Omega\right)}^{2}
$$

Proof.

$$
\begin{aligned}
\lambda_{j}+\lambda_{h, j} & =\lambda_{j}\left\|u_{j}\right\|_{L^{2}(\Omega)}^{2}+\lambda_{h, j}\left\|u_{h, j}\right\|_{L^{2}(\Omega)}^{2} \\
& =a\left(u_{j}, u_{j}\right)+a_{h}\left(u_{h, j} \cdot u_{h, j}\right)-\lambda_{h, j}\left\|u_{h, j}\right\|_{L^{2}\left(\Omega_{h} \backslash \Omega\right)}^{2} \\
& =a\left(u_{j}-u_{h, j}, u_{j}-u_{h, j}\right)+2 a\left(u_{j}, u_{h, j}\right)+\left\|\nabla u_{h, j}\right\|_{L^{2}\left(\Omega_{h} \backslash \Omega\right)}^{2}-\lambda_{h, j}\left\|u_{h, j}\right\|_{L^{2}\left(\Omega_{h} \backslash \Omega\right)}^{2} \\
& =\left\|\nabla\left(u_{j}-u_{h, j}\right)\right\|_{L^{2}(\Omega)}^{2}+2 \lambda_{j} \int_{\Omega} u_{j} u_{h, j}+\left\|\nabla u_{h, j}\right\|_{L^{2}\left(\Omega_{h} \backslash \Omega\right)}^{2}-\lambda_{h, j}\left\|u_{h, j}\right\|_{L^{2}\left(\Omega_{h} \backslash \Omega\right)}^{2} \\
& =\left\|\nabla\left(u_{j}-u_{h, j}\right)\right\|_{L^{2}(\Omega)}^{2}+2 \lambda_{j}-\lambda_{j}\left\|u_{j}-u_{h, j}\right\|_{L^{2}(\Omega)}^{2}+\left\|\nabla u_{h, j}\right\|_{L^{2}\left(\Omega_{h} \backslash \Omega\right)}^{2}-\lambda_{h, j}\left\|u_{h, j}\right\|_{L^{2}\left(\Omega_{h} \backslash \Omega\right)}^{2},
\end{aligned}
$$

then (4.18) holds.

Theorem 4.2. Let $\lambda_{j}$ be the $j$-th (simple) eigenvalue of problem (1.2) and let $\lambda_{h, j}$ be the associated $j$-th eigenvalue solution of (1.3). Then, the corresponding eigenfunctions $u_{j}$ and $u_{h, j}$ can be chosen such that $\left\|u_{j}\right\|_{L^{2}(\Omega)}=1,\left\|u_{h, j}\right\|_{L^{2}(\Omega)}=1$ and

$$
\left\|u_{j}-u_{h, j}\right\|_{H^{1}(\Omega)} \leq C h \sqrt{\log (1 / h)}
$$

with $C$ a strictly positive constant.

Proof. From (4.18) we observe that

$$
\left\|\nabla\left(u_{j}-u_{h, j}\right)\right\|_{L^{2}(\Omega)}^{2} \leq\left|\lambda_{h, j}-\lambda_{j}\right|+\lambda\left\|u_{j}-u_{h, j}\right\|_{L^{2}(\Omega)}^{2}+\left\|\nabla u_{h, j}\right\|_{L^{2}\left(\Omega_{h} \backslash \Omega\right)}^{2}+\lambda_{h, j}\left\|u_{h, j}\right\|_{L^{2}\left(\Omega_{h} \backslash \Omega\right)}^{2}
$$

and so, the result follows from Corollary 4.1, Theorem 4.1 and inequalities (4.13) and (4.17).

\section{Numerical Experiments}

In this section we present numerical approximations of the first eigenvalue for the problem (1.2) showing an order of convergence in agreement with the theoretical results. On the other hand our numerical experiments suggest that the discrete eigenvalue sequence is monotonically decreasing.

Table 1 shows numerical approximations of the first eigenvalue for $\alpha=2$. Considering the reference value $\lambda=7.71$ obtained by extrapolation, Table 2 presents the relative error $\mid \lambda-$ $\lambda_{h, 1} \mid / \lambda$. Let us notice that, assuming that the error $\left|\lambda-\lambda_{h, 1}\right|$ behaves as $C h^{R} \log h$, we have $R \approx 2$ (as suggested by Theorem 4.1). Similar results are obtained for different values of $\alpha$, $1<\alpha<3$.

In all the cases computed we observe that the discrete sequence monotonically decreases with $h$. As it is shown in Table 1 for $\alpha=2$ and in Table 3 where we display the numerical result for $\alpha=1.5$. 


\begin{tabular}{|c|c|}
\hline number of nodes & $\lambda_{h, 1}$ \\
\hline 26 & 8.25716040850849 \\
87 & 7.74861922546067 \\
254 & 7.72269504278331 \\
884 & 7.71351132128358 \\
3447 & 7.71083240068189 \\
\hline
\end{tabular}

TABLE 1. Numerical approximation of the first eigenvalue for $\alpha=2$ with graded meshes

\begin{tabular}{|c|c|}
\hline number of nodes & $\left|\lambda-\lambda_{h, 1}\right| / \lambda$ \\
\hline 26 & 0.070967627562709 \\
87 & 0.005008978658972 \\
254 & 0.001646568454385 \\
884 & 0.000455424290996 \\
3447 & 0.000107963771970 \\
\hline
\end{tabular}

TABLE 2. Errors $\left|\lambda-\lambda_{h, 1}\right| / \lambda$ for the first eigenvalue for $\alpha=2$ with graded meshes

\begin{tabular}{|c|c|}
\hline number of nodes & $\lambda_{h, 1}$ \\
\hline 16 & 6.47453652207 \\
42 & 6.37127872165 \\
157 & 6.28202778561 \\
533 & 6.27324527028 \\
2113 & 6.26993965925 \\
\hline
\end{tabular}

TABLE 3. Numerical approximation of the first eigenvalue for $\alpha=1.5$ with graded meshes

Indeed, from (4.18), we have

$\lambda_{h, j}-\lambda_{j}=\frac{\left(\left\|\nabla\left(u_{j}-u_{h, j}\right)\right\|_{L^{2}(\Omega)}^{2}+\left\|\nabla u_{h, j}\right\|_{L^{2}\left(\Omega_{h} \backslash \Omega\right)}^{2}\right)-\lambda_{h, j}\left(\left\|u_{j}-u_{h, j}\right\|_{L^{2}(\Omega)}^{2}+\left\|u_{h, j}\right\|_{L^{2}\left(\Omega_{h} \backslash \Omega\right)}^{2}\right)}{1-\left\|u_{j}-u_{h, j}\right\|_{L^{2}(\Omega)}^{2}}$,

and for $h$ small the sign of $\lambda_{h, j}-\lambda_{j}$ depends on the difference of the two terms given in the numerator. Since we would expect the second one to be of higher order than the first one, we can conjecture that $\lambda_{h, j}-\lambda_{j}>0$ for $h$ small enough.

On the other hand, assuming $\lambda_{h, j}-\lambda_{j}>0$ and considering $\lambda_{H, j}$ and $\lambda_{h, j}$, eigenvalues of problem (1.3) with $H>h$, we can write $\lambda_{H, j}-\lambda_{h, j}=\lambda_{H, j}-\lambda_{j}-\left(\lambda_{h, j}-\lambda_{j}\right)$. Then, Theorem 4.1 would imply the monotonicity of the discrete sequence of eigenvalues.

Acknowledgments: We want to thank Rodolfo Rodríguez for several fruitful discussions and suggestions. 


\section{REFERENCES}

[1] G. Acosta and M. G. Armentano (2011), Finite element approximations in a non-Lipschitz domain: Part. II, Math. Comp. 80(276), pp. 1949-1978 .

[2] G. Acosta, M. G. Armentano, R. G. Durán and A. L. Lombardi (2005), Nonhomogeneous Neumann problem for the Poisson equation in domains with an external cusp, Journal of Mathematical Analysis and Applications 310(2), pp. 397-411.

[3] G. Acosta, M. G. Armentano, R. G. Durán and A. L. Lombardi (2007), Finite element approximations in a non-Lipschitz domain, SIAM J. Numer. Anal. 45(1), pp. 277-295.

[4] I. Babuska and J. Osborn (1991), Eigenvalue Problems, Handbook of Numerical Analysis, vol. II, Finite Element Methods (Part.1).

[5] D. Boffi (2010), Finte element aproximation of eigenvalue problems, Acta Numerica 19, pp. 1-120.

6] P. Grisvard (1985), Elliptic Problems in Nonsmooth Domains, Pitman, Boston.

[7] E. Hernández And R. Rodríguez (2004), Finite Element Approximation of Spectral Problems with Neumann Boundary Conditions on Curved Domains, Math. Comp. 72(243), pp. 1099-1115.

[8] A. Khelif (1978), Equations aux derivees partiellles, C.R. Acad. Sc. Paris 287, pp. 1113-1116.

[9] M.P. Lebaud (1994), Error estimate in an isoparametric finite element eigenvalue problem, Math. Comp. 63(207), pp. 19-40.

[10] V. Maz'ya, S. Poвоrchi (1997), Differentiable functions on bad domains, World Sci., Singapore.

[11] J. Osborn, (1975) Spectral Approximation for Compact Operators, Math. Comp. 29, pp. 712-725.

[12] M. VAnmaele And A. ŽEníšeK (1993), External finite element approximations of eigenvalue problems, $\mathrm{M}^{2} \mathrm{AN} 27(5)$, pp. 565-589.

[13] M. VAnMAele AND A. Z̆EnÍS̆EK (1994), External finite-element approximations of eigenfunctions in the case of multiple eigenvalues, J. Comput. Appl. Math. 50(1-3), pp. 51-66.

[14] M. Vanmaele And A. ŽEnís̆EK (1995), The combined effect of numerical integration and approximation of the boundary in the finite element methods for the eigenvalue problems, Numer. Math. 71(2), pp. 253 - 273.

Departamento de Matemática, Facultad de Ciencias Exactas y Naturales, Universidad de Buenos Aires, IMAS-Conicet, 1428 Buenos Aires, Argentina.

E-mail address: gacosta@dm.uba.ar

Departamento de Matemática, Facultad de Ciencias Exactas y Naturales, Universidad de Buenos Aires, imas-Conicet, 1428 Buenos Aires, Argentina.

E-mail address: garmenta@dm.uba.ar 